\title{
CALIPSO: Global aerosol and cloud observations from lidar and passive instruments
}

\author{
L. R. Poole ${ }^{* a}$, D. M. Winker**a , J. R. Pelon ${ }^{\sharp b}$, M. P. McCormick ${ }^{\# \# c ~}$

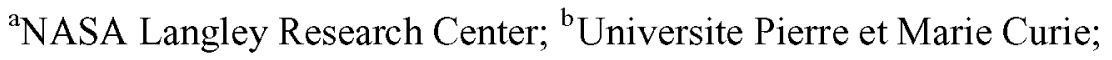 \\ 'Hampton University
}

\begin{abstract}
CALIPSO (Cloud-Aerosol Lidar and Infrared Pathfinder Spaceborne Observations) is an approved satellite mission being developed through collaboration between NASA and the French space agency CNES. The mission is scheduled for launch in 2004 and will operate for 3 years as part of a five-satellite formation called the Aqua constellation. This constellation will provide a unique data set on aerosol and cloud optical and physical properties and aerosol-cloud interactions that will substantially increase our understanding of the climate system and the potential for climate change.
\end{abstract}

Keywords: Lidar, Aerosol, Cloud, Radiative Forcing, Climate

\section{INTRODUCTION}

Uncertainties in the effects of clouds and aerosols on the Earth's radiation budget limit our understanding of the climate system and the potential for global climate change. CALIPSO (Cloud-Aerosol Lidar and Infrared Pathfinder Spaceborne Observations) is an approved satellite mission within the NASA Earth System Science Pathfinder (ESSP) program that will address these uncertainties through a combination of lidar and selected passive instruments. The mission (formerly known as PICASSO-CENA) is being developed through collaboration between NASA and the French space agency CNES for a launch in 2004, followed by 3 years of on-orbit operations.

The potential of spaceborne lidar for cloud and aerosol studies was demonstrated by The Lidar In-space Technology Experiment $^{1}$ (LITE), which flew on the Space Shuttle in September 1994. The CALIPSO lidar is a two-wavelength polarization-sensitive system that will provide data on the vertical distributions of aerosols and clouds and cloud ice/water phase (through the ratio of signals in two orthogonal polarization channels), and a qualitative classification of aerosol size (through the wavelength dependence of the backscatter). The CALIPSO payload also includes passive imagers operating in the visible and infrared spectral regions to provide additional information on aerosol and cloud optical and physical properties, overall meteorological context, and spatial co-registration of data.

CALIPSO is co-manifested with the ESSP CloudSat satellite for launch on a Delta II rocket. They both will be flown in a 705-km, circular polar orbit as part of the Aqua constellation, which also includes the NASA EOS (Earth Observing System) Aqua and Aura platforms and the CNES PARASOL (Polarization and Anisotropy of Reflectances for Atmospheric Science coupled with Observations from a Lidar) satellite. The constellation will collect a comprehensive suite of nearly coincident measurements of atmospheric state, aerosol and cloud optical properties, and radiative fluxes. This combined dataset will allow fundamental advances in our understanding of the links between aerosols, clouds, and radiation necessary to assess future climate change more accurately.

*1.r.poole@larc.nasa.gov; phone 1757 864-2689; fax 1757 864-7775; NASA Langley Research Center, MS 435, Hampton, VA 23681-2199 USA; **d.m.winker@larc.nasa.gov; phone 1757 8646747; fax 1757 864-7775; NASA Langley Research Center, MS 435, Hampton, VA 23681-2199 USA; \# jacques.pelon@aero.jussieu.fr; phone 331442737 79; fax 331442737 76; Institut Pierre Simon Laplace, Universite Pierre et Marie Curie, 4 place Jussieu, 75252 Paris cedex 05, FRANCE; " pat.mccormick@hamptonu.edu, phone 1757 728-6867; fax 1757 727-5090; Center for Atmospheric Sciences, Hampton University, Hampton, VA 23668 USA 


\section{SCIENCE OBJECTIVES}

\subsection{Aerosol radiative forcing}

Climate models indicate that aerosols can significantly impact the Earth's radiation budget, representing a so-called radiative forcing of climate. There is a direct radiative forcing due to absorption and scattering of solar radiation and absorption and emission of infrared radiation. Aerosols also affect the radiation budget indirectly by modifying the reflectance and lifetime of clouds through their role as cloud condensation nuclei. Figure 1 shows recent ${ }^{2}$ estimates of the global average radiative forcing since the year 1750 due to changes in well-mixed greenhouse gases $\left(\mathrm{CO}_{2}, \mathrm{CH}_{4}, \mathrm{~N}_{2} \mathrm{O}\right.$, and halocarbons) and tropospheric aerosols. The bars in the graph denote estimates of mean forcing, while the capped vertical lines denote the range of uncertainty about the mean. The vertical lines with " 0 " delimiters indicate aerosol forcings for which no estimate of the mean can be made due to the large uncertainties.

The estimates of aerosol radiative forcing are highly uncertain because they are based primarily on model calculations, and current capabilities for observing aerosols from space are inadequate to constrain key assumptions in these models. Another complicating factor is that tropospheric aerosols, unlike greenhouse gases, are highly variable in space and time due to variable sources and short atmospheric residence times. As a consequence, regional aerosol radiative forcings can be much larger than the global mean values depicted in the figure. The CALIPSO mission will enhance current capabilities by providing near-global vertically resolved measurements of aerosol distribution, an ability to perform height-resolved discrimination of aerosols into several types, and an improved capability to observe aerosols over bright and heterogeneous surfaces.

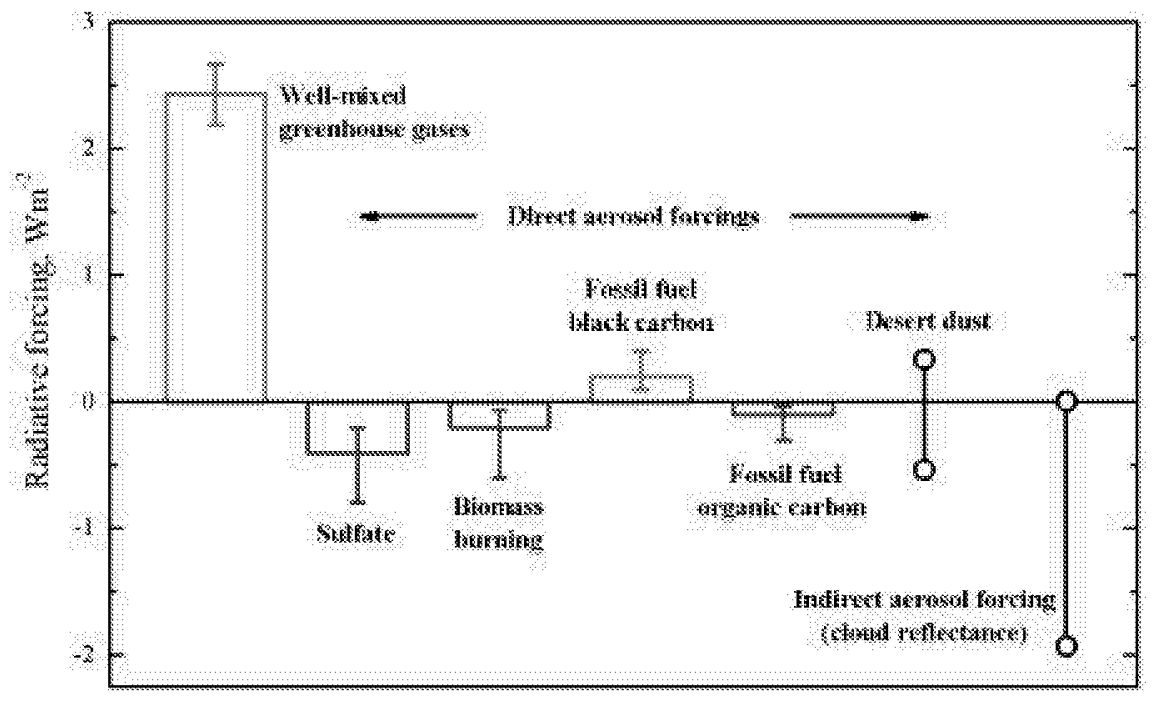

Figure 1. Global mean radiative forcing (bars) since 1750 due to changes in well-mixed greenhouse gases and aerosols ${ }^{2}$, with uncertainty ranges indicated by vertical lines.

\subsection{Cloud-radiation interactions}

The sensitivity of the climate to external forcings such as those imposed by greenhouse gases and aerosols is largely controlled by interactions between clouds and radiation. Advances in model capabilities to predict climate change requires improved represent ations of cloud processes and cloud-radiation interactions in those models. The fundamental problem lies in accurately modeling the cloud feedback loop shown in Figure 2. The largest uncertainties involve the use of models to (a) predict cloud properties based on knowledge of atmospheric state, and (b) use of these cloud properties to calculate radiative energy fluxes. In particular, the largest source of uncertainty in estimating infrared 
radiative fluxes at the surface and within the atmosphere are connected with current difficulties in determining the vertical distribution and overlap of multi-layer clouds and their ice-water path.

Because of the short time scales and nonlinear relationships typical of cloud processes, nearly simultaneous observations of atmospheric state, aerosol and cloud optical properties, and radiative fluxes are necessary to test the ability of cloud models to reproduce the physics of cloud-radiation feedbacks. The five satellites of the Aqua constellation will provide an unprecedented, comprehensive suite of nearly coincident measurements of atmospheric state, aerosol and cloud optical properties, and radiative fluxes. This combined dataset will allow fundamental advances in our understanding of the links between aerosols, clouds, and radiation necessary to more accurately assess future climate change.

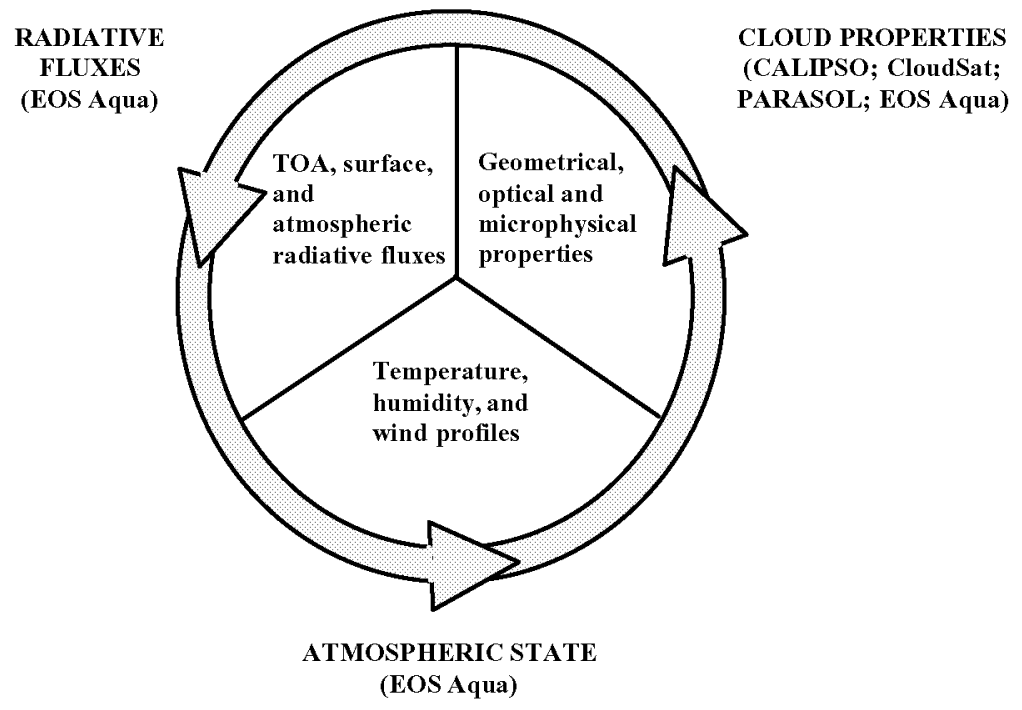

Figure 2. Measurements of the cloud-radiation feedback loop to be provided by satellites in the Aqua constellation.

\section{CALIPSO PAYLOAD}

The CALIPSO payload consists of three co-aligned, nadir-viewing instruments: the Cloud-Aerosol LIdar with Orthogonal Polarization (CALIOP), the Imaging Infrared Radiometer (IIR), and the Wide Field Camera (WFC). These instruments are designed to operate autonomously and continuously, although the WFC acquires science data only under daylight conditions. Science data are downlinked using an X-band transmitter system that is part of the payload. The layout of the instruments on the payload is shown in Figure 3.

\subsection{CALIOP}

The lidar transmitter subsystem consists of two redundant diode-pumped Nd:YAG lasers, each with a beam expander and a beam-steering system. The lasers produce $110 \mathrm{~mJ}$ of energy at each of the two operating wavelengths (1064 $\mathrm{nm}$ and $532 \mathrm{~nm}$ ) at a pulse repetition rate of $20.2 \mathrm{~Hz}$. Beam expanders reduce the angular divergence of the laser beam to produce a beam diameter at the Earth's surface of 70 meters. The lasers are passively cooled using a dedicated radiator panel, avoiding the use of pumps and coolant loops. Each laser is housed in its own sealed canister filled with dry air at standard atmospheric pressure.

A space-qualifiable prototype laser (called the Risk Reduction Laser, or RRL) designed to meet all performance requirements of the flight lasers was developed and tested to reduce risk in the development of the flight lasers. The 
RRL was subjected to an extended life test that began in September 1998 and concluded in August 2001. During the test program, the RRL was operated for more than 2 billion shots (equivalent to a 3 -year mission lifetime) without significant degradation. This test program has validated the laser design and the contamination control procedures developed for the flight lasers.

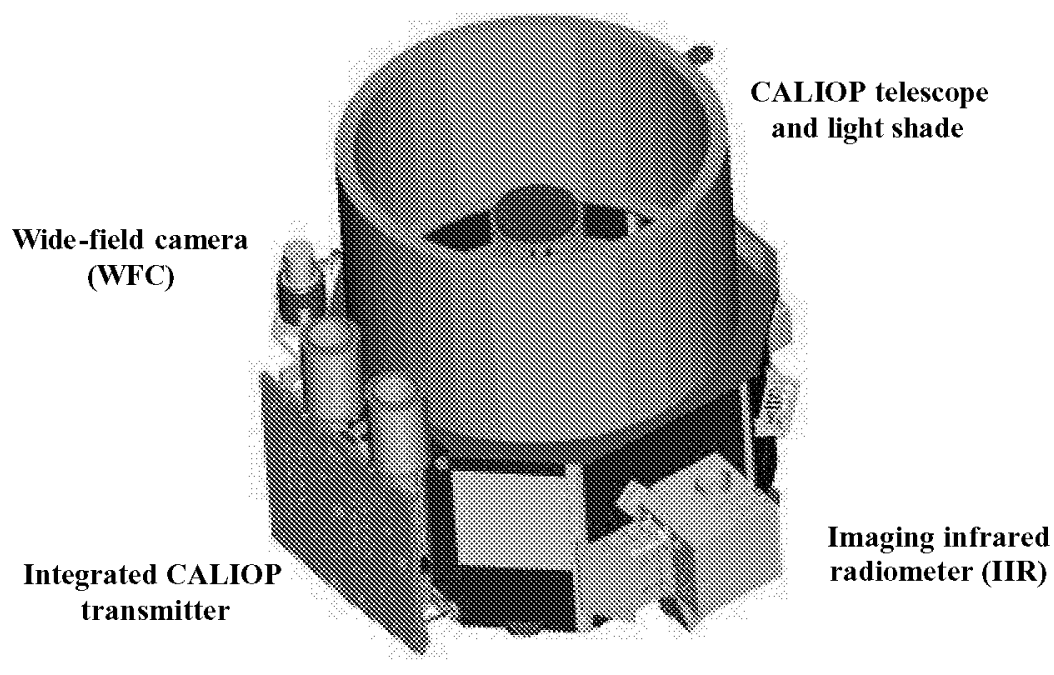

Figure 3. Layout of the CALIPSO payload.

CALIOP has three receiver channels: one measuring the 1064-nm backscattered intensity, and two channels measuring orthogonally polarized components (parallel and perpendicular to the polarization plane of the transmitted beam) of the 532-nm backscattered signal. The receiver telescope is 1 meter in diameter, with the primary mirror, secondary mirror, metering structure, and inner baffle all made of beryllium. A carbon composite light shade prevents direct solar illumination of the mirrors. The full-angle field of view of the telescope is 130 microradians, resulting in a footprint at the Earth's surface (from a 705-km orbit) of about 90 meters. A mechanism located in the collimated portion of the beam contains a shutter and a depolarizer used in calibrating the $532-\mathrm{nm}$ perpendicular channel. A narrow-band (35 picometer) etalon is used to reduce the solar background illumination at $532 \mathrm{~nm}$, while a broader ( 0.4 nanometer) dielectric interference filter provides sufficient solar rejection for the 1064-nm channel. Dual digitizers on each channel provide the dynamic range needed to measure backscatter signals from both clouds and the molecular atmosphere. An active boresight system is used to maintain co-alignment between the transmitter and the receiver.

The fundamental sampling resolution of CALIOP is 30 meters in altitude and 1/3 kilometer along track. Backscatter data will be acquired at this resolution from the Earth's surface to $40 \mathrm{~km}$, and will be downlinked at full resolution in the lower troposphere. Data from higher altitudes will be averaged onboard to meet telemetry bandwidth limitations. Data profiles will be averaged to $60-\mathrm{m}$ vertical resolution and $1-\mathrm{km}$ horizontal resolution in the upper troposphere, and to 180$\mathrm{m}$ vertical resolution and $5-\mathrm{km}$ horizontal resolution in the stratosphere. CALIOP profiles are calibrated by normalizing the backscattered signal between $30-35 \mathrm{~km}$ to the signal expected in that altitude range from the molecular atmosphere. Onboard hardware is used to calibrate the $532-\mathrm{nm}$ perpendicular channel relative to the $532-\mathrm{nm}$ parallel channel. The $1064-\mathrm{nm}$ channel is calibrated relative to the $532-\mathrm{nm}$ total backscatter signal using cirrus clouds as targets.

A detailed simulation computer model has been developed to assess CALIOP performance and test retrieval algorithms. Simulated CALIPSO raw data derived from LITE observations along a $1500-\mathrm{km}$ orbit segment over western Africa are shown in Figure 4. Because the LITE instrument did not measure depolarization, clouds above about $10 \mathrm{~km}$ in altitude were prescribed to be ice in this simulation, with particle depolarization of $30 \%$. Clouds below about $10 \mathrm{~km}$, aerosols, and the Earth's surface are prescribed to be non-depolarizing. Note that even the layer of thin, subvisible cirrus near 17 $\mathrm{km}$ altitude stands out clearly in both parallel and perpendicular $532-\mathrm{nm}$ channels. The simulated backscatter signal at 
$1064 \mathrm{~nm}$ is somewhat noisier than the $532-\mathrm{nm}$ signal, but the cloud and aerosol layers are still clearly discernable relative to the background molecular atmosphere.
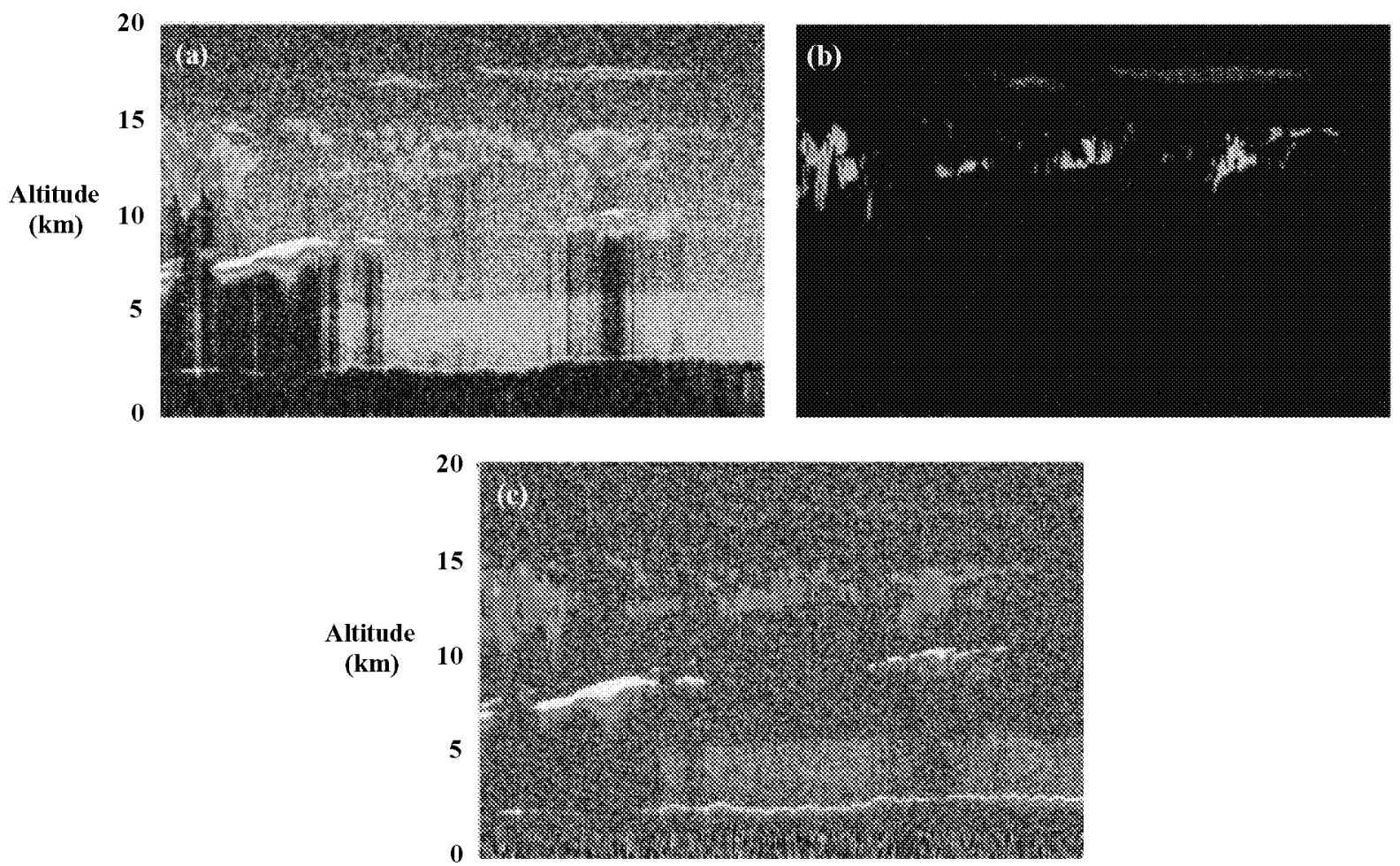

Figure 4. Simulated CALIOP aerosol and cloud data: (a) 532-nm parallel polarization channel; (b) 532-nm perpendicular polarization channel; (c) 1064-nm channel

\subsection{IIR}

The IIR (imaging infrared radiometer) is being provided by CNES, with algorithm development being performed by the Institute Pierre Simon Laplace (Paris). It is a nadir-viewing, non-scanning imager having a $64 \mathrm{~km}$ by $64 \mathrm{~km}$ swath with a pixel size of $1 \mathrm{~km}$. The CALIOP beam is nominally aligned with the center of an IIR image. The IIR uses a single microbolometer detector array, with a rotating filter wheel providing measurements at three channels in the window region, on either side of the $\mathrm{O}_{3}$ absorption band at $9.6 \mu \mathrm{m}$. The central wavelengths (and spectral widths) of the three channels are $8.65 \mu \mathrm{m}(0.9 \mu \mathrm{m}), 10.6 \mu \mathrm{m}(0.6 \mu \mathrm{m})$, and $12.05 \mu \mathrm{m}(1.0 \mu \mathrm{m})$. These wavelengths were selected to optimize joint CALIOP/IIR retrievals of cirrus cloud emissivity and particle size.

On-orbit calibration of the IIR is performed to relate the radiometric measurements to an absolute standard in each spectral channel. The calibration procedure requires viewing scenes at two different known temperatures. A cold scene calibration $(4 \mathrm{~K})$ is achieved by observing deep space every 8 seconds, while a hot scene calibration $(300 \mathrm{~K})$ is performed every 40 seconds using an internal blackbody source. 


\subsection{WFC}

The WFC (wide-field camera) is a modified version of the commercial off-the-shelf (COTS) Ball Aerospace CT-633 star-tracker camera. It is a fixed, nadir viewing imager with a single spectral channel covering the $620-670 \mathrm{~nm}$ region, which was selected to match band 1 of the MODIS (MODerate resolution Imaging Spectroradiometer) instrument on EOS Aqua. The WFC is operated in a push-broom mode, collecting images with 125-meter spatial resolution over a 61$\mathrm{km}$ cross-track swath centered on the CALIOP beam. The WFC provides meteorological context for the CALIOP data and enables highly accurate spatial registration, when required, between measurements from CALIPSO and other instruments of the Aqua constellation. WFC images will be also used to assess cloud fraction within 1-km IIR pixels to enhance the retrieval of cloud properties from the IIR data. Figure 5 shows examples of simulated images from the WFC and from the 10.6- $\mu \mathrm{m}$ channel of the IIR.

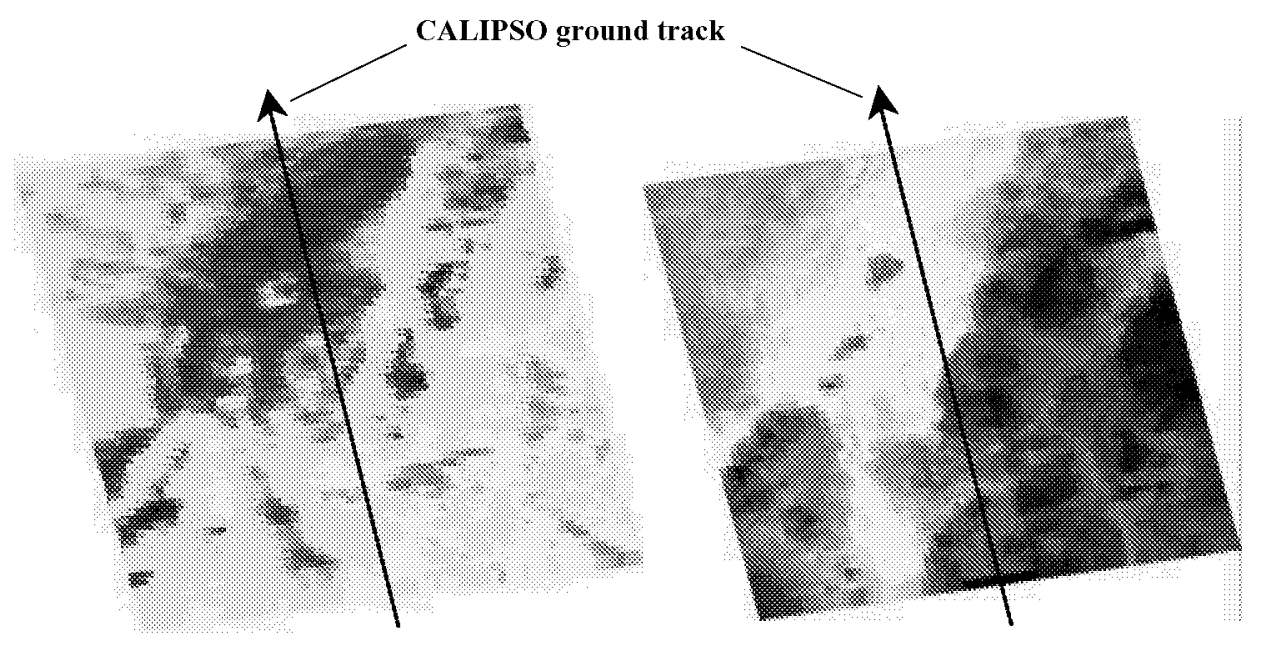

Figure 5. Simulated images from the IIR 10.6- $\mu \mathrm{m}$ channel (left) and the WFC (right) of a dry lakebed in northern Mexico.

\subsection{Data products}

The primary data products of CALIPSO and the instruments used to derive those products are listed in Table 1.

\begin{tabular}{|l|c|c|c|}
\hline \multicolumn{1}{|c|}{ Measurement } & CALIOP & WFC & IIR \\
\hline Aerosol profiles & $\bullet$ & & \\
\hline Aerosol optical depth & $\bullet$ & & \\
\hline Cloud height and thickness & $\bullet$ & & \\
\hline Cloud optical depth & $\bullet$ & & \\
\hline Cloud ice/water phase & $\bullet$ & & \\
\hline Cirrus emissivity & $\bullet$ & $\bullet$ & $\bullet$ \\
\hline Cirrus particle size & $\bullet$ & $\bullet$ & $\bullet$ \\
\hline
\end{tabular}

Table 1. CALIPSO aerosol and cloud measurements. 


\subsection{Current status}

The CALIPSO payload is scheduled for delivery in July 2003. The composite payload structure, including the basic housing, the optical bench, and the light shade for the telescope is complete, as are the telescope and the laser optics module. All of the optical components for the CALIOP receiver have been received and are now being integrated onto the optical bench and aligned. The photomultiplier tubes for the CALIOP 532-nm parallel and perpendicular channels are complete and are undergoing detailed characterization tests. CALIOP components still in assembly or test include the avalanche photodiode for the 1064-nm channel, the receiver electronics, the depolarizer mechanism used for relative calibration of the 532-nm polarization channels, and the boresight mechanism that maintains alignment between the transmitted laser beam and telescope. The IIR is presently in the integration stage, with qualification tests beginning in mid-October 2002 and calibration tests scheduled for mid-December. Delivery of the IIR is expected at the end of January 2003. All WFC hardware modifications are complete and software modifications are in the testing stage, with WFC delivery expected at the end of August 2002. Pictures of selected payload components are shown in Figure 6.
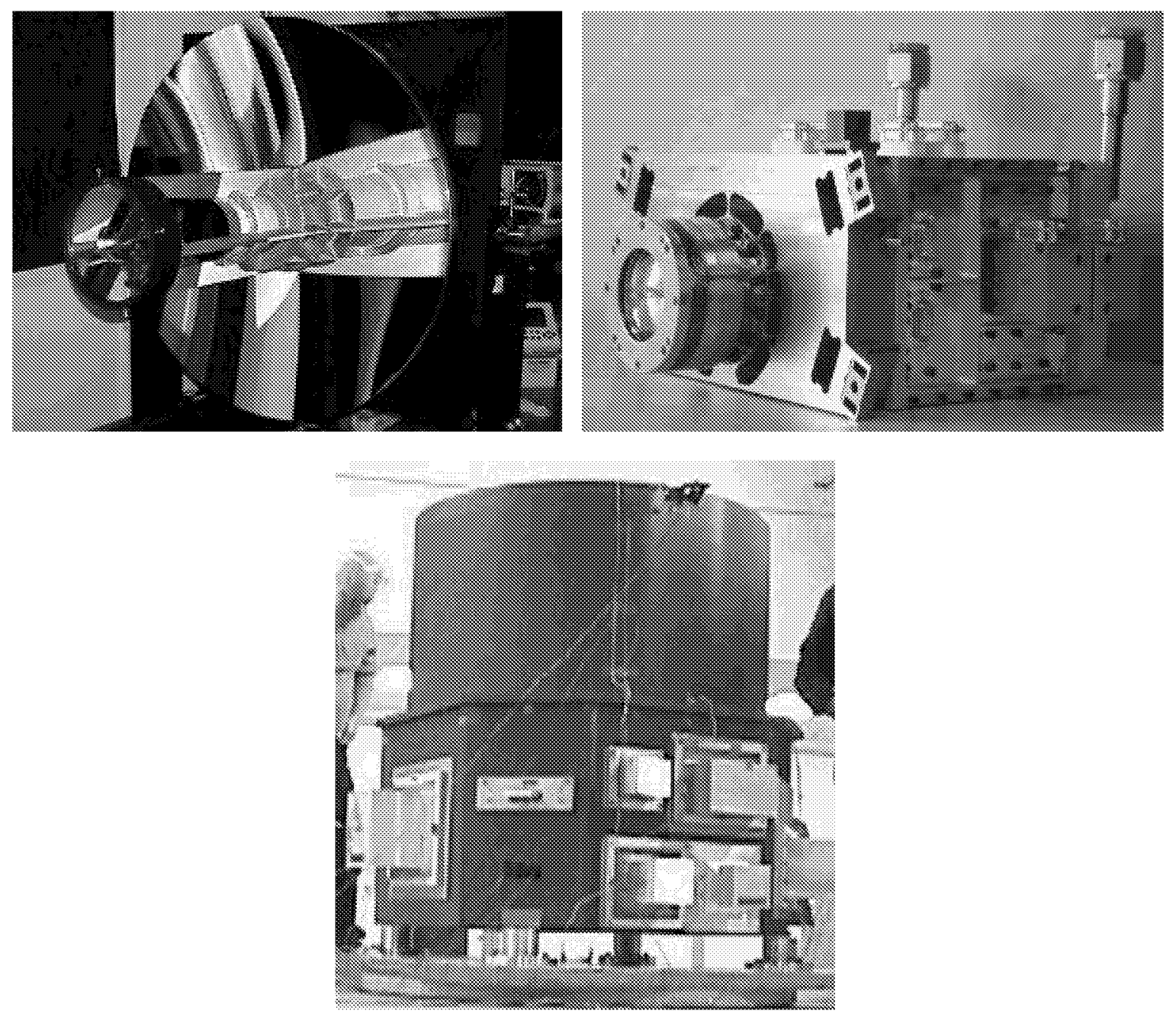

Figure 6. Photographs of selected CALIPSO payload components. Top left: telescope; top right: IIR infrared sensor module; bottom: payload structure and telescope light shade. 


\section{AQUA CONSTELLATION MEASUREMENT SYNERGIES}

The satellites of the Aqua constellation will fly in a sun-synchronous, $705-\mathrm{km}$ circular orbit with nominal ascending node equatorial crossing times ranging from 13:30 local (EOS Aqua) to 13:45 local (EOS Aura). The orbits of CALIPSO, CloudSat, and PARASOL will be maintained (Figure 6) to provide measurements that are nearly coincidental in space and time with those from Aqua, which was launched in May 2002. Instruments on Aqua will collect a variety of data on aerosols, clouds, atmospheric state, and atmospheric radiation. MODIS will provide the geographic distribution of aerosol optical depth and particle size, cloud optical depth, droplet size, and liquid water path. CERES (Clouds and the Earth's Radiant Energy System) will provide broadband radiative fluxes at the top of the atmosphere, at the Earth's surface, and throughout the atmospheric column. AIRS (Atmospheric InfraRed Sounder) and HSB (Humidity Sounder for Brazil) will provide atmospheric temperature and humidity profiles, respectively, and AMSR-E (Advanced Microwave Scanning Radiometer-EOS) will provide estimates of cloud height, phase, and liquid water path.

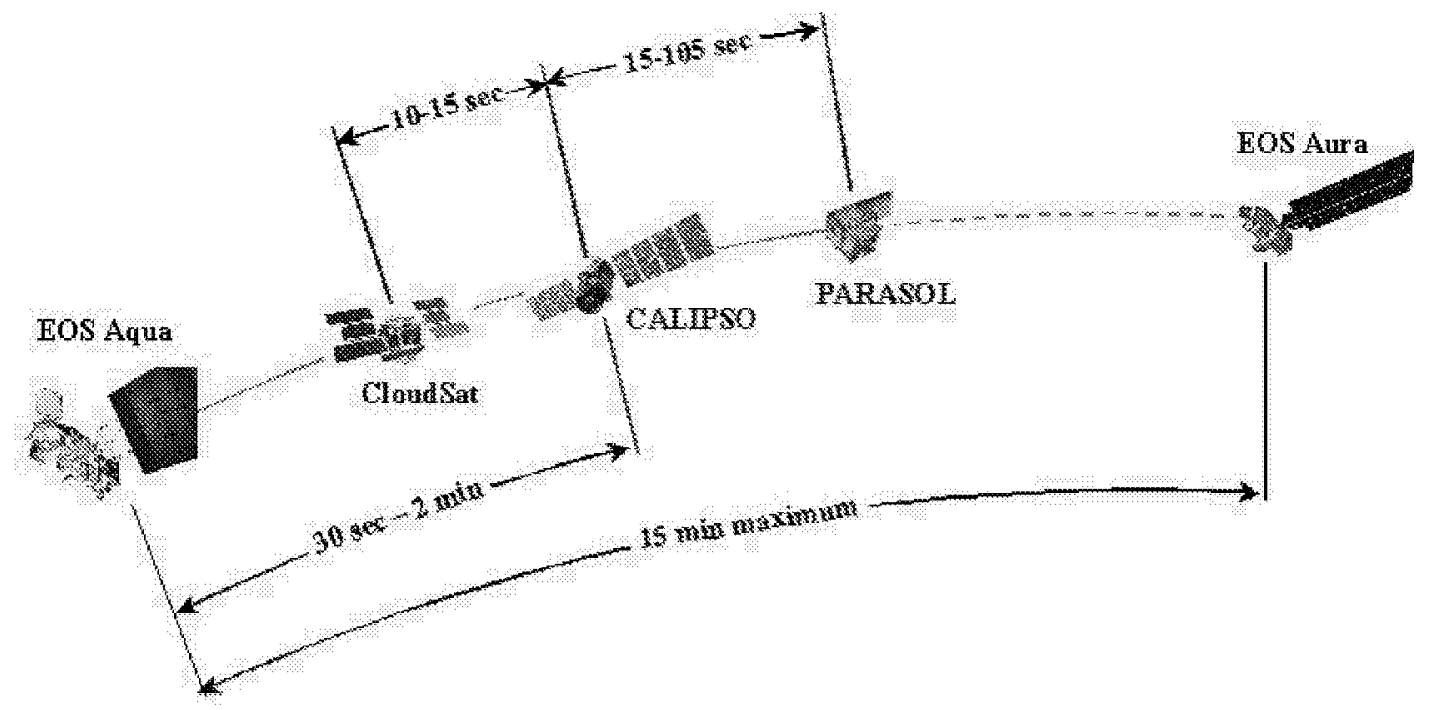

Figure 7. The five satellites of the EOS Aqua constellation.

CloudSat will be launched with CALIPSO and will fly a 94-GHz radar to provide profiles of the optical and physical properties of thick clouds that CALIOP cannot fully penetrate, as well as information on drizzle below liquid clouds. The OMI (Ozone Monitoring Instrument) on EOS Aura will provide information on the geographic distribution of absorbing aerosols. PARASOL, which will be launched 9 months after the launch of CALIPSO, will provide unique information on aerosol and cloud microphysical and radiative properties using a multi-channel, wide field-of-view, polarization-sensitive camera. Each satellite in the constellation offers unique information on clouds and aerosols, and combining their data will provide greater insight on the roles of these important constituents in the climate system than could be gained from any single satellite.

\section{REFERENCES}

1. D. M. Winker, M. P. McCormick, and R. Couch, "An overview of LITE: NASA's Lidar In-space Technology Experiment," Proc. IEEE 84, pp. 164-180, 1996.

2. IPCC, 2001: Climate Change, 2001: The Scientific Basis. Contribution of Working Group I to the Third Assessment Report of the Intergovernmental Panel on Climate Change [J. T. Houghton, Y. Ding, D. J. Griggs, M. Noguer, P. J. van der Linden, X. Dai, K. Maskell, and C. A. Johnson (eds.)], Cambridge University Press, Cambridge, United Kingdom and New York, NY, USA, 881 pp. 